Pacific

Journal of

Mathematics

THE MODULE OF DERIVATIONS FOR AN ARRANGEMENT OF SUBSPACES

JONATHAN WIENS

Volume $198 \quad$ No. 2

April 2001 


\title{
THE MODULE OF DERIVATIONS FOR AN ARRANGEMENT OF SUBSPACES
}

\author{
JONATHAN WIENS
}

\begin{abstract}
This paper examines the module of derivations for a subspace arrangement. In particular, we consider those subspace arrangements consisting of elements of the intersection lattice of a generic hyperplane arrangement. We determine generators for the associated module of derivations. These generators are indexed by certain elements of the intersection lattice.
\end{abstract}

\section{Introduction.}

Let $V$ be a linear space of dimension $\ell$ over a field $\mathbf{K}$. By an arrangement we shall mean a finite collection of affine subspaces of $V$. If all of the subspaces in an arrangement $\mathcal{A}$ have codimension $k$ then we say that $\mathcal{A}$ is an $(\ell, k)$ arrangement. If $k=1$ and so $\mathcal{A}$ is a hyperplane arrangement then we shall say that $\mathcal{A}$ is an $\ell$-arrangement.

Let $\mathcal{A}$ be an arrangement and $S$ the coordinate ring for $V$. For each $\mathrm{H} \in \mathcal{A}$ let $I_{\mathrm{H}}=\mathrm{V}(\mathrm{H})$, the ideal of $S$ which vanishes on $\mathrm{H}$, and call it the defining ideal for $\mathrm{H}$. If $\mathrm{H}$ is a hyperplane, then we can choose a linear functional $\alpha_{\mathrm{H}} \in S$ such that $I_{\mathrm{H}}=\left(\alpha_{\mathrm{H}}\right)$.

We now introduce the main character of this paper. If $\mathcal{A}$ is an arrangement then the module of $\mathcal{A}$-derivations is $\mathrm{D}(\mathcal{A})$, the set of all $\mathbf{K}$-linear derivations of $S$ which map each defining ideal to itself. Equivalently, one could define $\mathrm{D}(\mathcal{A})$ to be the set of all polynomial vector fields which, at each subspace, are parallel to that subspace. [1] contains an extensive review of the properties of $\mathrm{D}(\mathcal{A})$ for hyperplane arrangements, especially for free arrangements. We shall review the situation for generic arrangements in Section 3.

Recently interest has arisen in arrangements of subspaces of codimension greater than one. The goal of this paper is to examine $\mathrm{D}(\mathcal{A})$ in this case. In particular we investigate subspace arrangements consisting of elements of the intersection lattice of a generic hyperplane arrangement, where we find generators for $\mathrm{D}(\mathcal{A})$ as an $S$-module.

In Section 2 we list several elementary properties of $\mathrm{D}(\mathcal{A})$. In Section 3 we find generators of $\mathrm{D}(\mathcal{A})$ for generic arrangements. In Section 4 we discuss 
subspace arrangements arising from hyperplane arrangements and in Section 5 we find generators for $\mathrm{D}(\mathcal{A})$ for those subspace arrangements arising from generic hyperplane arrangements.

The author would like to thank Sergey Yuzvinsky for valuable suggestions during the preparation of this paper.

\section{Subspace Arrangements.}

In this section we define our terminology and give some elementary results. Let $\mathcal{A}=\left\{\mathrm{H}_{1}, \ldots, \mathrm{H}_{n}\right\}$ be an arrangement with defining ideals $\left\{I_{\mathrm{H}_{1}}, \ldots\right.$, $\left.I_{\mathrm{H}_{n}}\right\}$. An element $\mathrm{H}$ of $\mathcal{A}$ is maximal if it is not contained in any other subspace of $\mathcal{A}$. $\mathcal{A}$ is central if $T=\cap_{i=1}^{n} \mathrm{H}_{i}$ is non-empty. In this case we choose coordinates so that $0 \in T$. We say that $\mathcal{A}$ is essential if $T=0$. We shall primarily concern ourselves with central arrangements.

Let $S$ be the coordinate ring for $V$. Let $\operatorname{Der}_{\mathbf{K}}(S)$ denote the set of $\mathbf{K}$ linear derivations of $S$, that is, K-linear maps $\theta: S \rightarrow S$ such that $\theta(f g)=$ $f \theta(g)+g \theta(f)$ for all $f, g \in S$.

Definition 2.1. Let $\mathcal{A}$ be an arrangement in $V$. The module of $\mathcal{A}$-derivations is

$$
\mathrm{D}(\mathcal{A})=\left\{\theta \in \operatorname{Der}_{\mathbf{K}}(S) \mid \theta\left(I_{\mathrm{H}}\right) \subseteq I_{\mathrm{H}} \forall \mathrm{H} \in \mathcal{A}\right\} .
$$

One obvious consequence of the definition of $\mathrm{D}(\mathcal{A})$ is the following lemma.

Lemma 2.2. If $\mathcal{B} \subseteq \mathcal{A}$ are a pair of arrangements then $\mathrm{D}(\mathcal{A}) \subseteq \mathrm{D}(\mathcal{B})$.

If $\mathcal{A}$ is central, then the defining ideals of $\mathcal{A}$ are all homogeneous with degree one generators and hence $\mathrm{D}(\mathcal{A})$ is a graded $S$-module. In this case let $V^{*}$ denote the dual space of the vector space $V$ and $S^{+}$denote the maximal graded ideal of $S$. We shall abbreviate $\frac{\partial}{\partial x_{i}}$ by $D_{i}$. The Euler derivation is $\theta_{E}=\sum_{i=1}^{\ell} x_{i} D_{i}$ and has the property that if $f \in S$ is homogeneous of degree $n$ then $\theta_{E}(f)=n f$. As a result we have:

Lemma 2.3. If $\mathcal{A}$ is a central arrangement then $\theta_{E} \in \mathrm{D}(\mathcal{A})$.

The Euler derivation plays a deeper role in some arrangements.

Lemma 2.4. If $\mathcal{A}$ is central and contains a hyperplane then $S \theta_{E}$ is a direct summand of $\mathrm{D}(\mathcal{A})$.

Proof. If $\mathrm{H} \in \mathcal{A}$ is a hyperplane choose a linear functional $\alpha \in V^{*}$ so that $\mathrm{H}=V(\alpha)$. If $\theta \in \mathrm{D}(\mathcal{A})$ then $\alpha$ divides $\theta(\alpha)$ and hence we can define the function $\phi: \mathrm{D}(\mathcal{A}) \rightarrow S$ by $\phi(\theta)=\theta(\alpha) / \alpha$. Since $\theta_{E} \in \mathrm{D}(\mathcal{A}), \phi$ is surjective with section $s: S \rightarrow \mathrm{D}(\mathcal{A})$ given by $s(f)=f \theta_{E}$. This shows $S \theta_{E}$ is a direct summand of $\mathrm{D}(\mathcal{A})$.

While freeness is an important property for hyperplane arrangements, it rarely occurs in more general arrangements. 
Theorem 2.5. If $\mathcal{A}$ contains a maximal subspace of codimension greater than 1 , then $\mathrm{D}(\mathcal{A})$ is not a free $S$-module.

Proof. Suppose that $\mathrm{D}(\mathcal{A})$ is free, with basis $\theta_{1}, \ldots, \theta_{m}$. Let $\mathrm{H} \in \mathcal{A}$ be maximal with codimension $k>1$. Choose a basis $\left\{x_{1}, \ldots, x_{\ell}\right\}$ for $V$ so that $I_{\mathrm{H}}=\left(x_{1}, \ldots, x_{k}\right)$. Since $\mathrm{H}$ is maximal, for each $\mathrm{K} \neq \mathrm{H}$ in $\mathcal{A}$ there exists $\beta_{\mathrm{K}} \in I_{\mathrm{K}}$ such that $\beta_{\mathrm{K}} \notin I_{\mathrm{H}}$. Let $\beta=\prod\left\{\beta_{\mathrm{K}} \mid \mathrm{K} \neq \mathrm{H}\right\}$ and note that $\beta \notin I_{\mathrm{H}}$. Hence $\beta D_{1} \notin \mathrm{D}(\mathcal{A})$, but $x_{1} \beta D_{1}, x_{2} \beta D_{1} \in \mathrm{D}(\mathcal{A})$. Now $x_{1} \beta D_{1}=\sum_{i=1}^{m} p_{i} \theta_{i}$ and $x_{2} \beta D_{1}=\sum_{i=1}^{m} q_{i} \theta_{i}$ and hence $x_{2} p_{i}=x_{1} q_{i}$ for all $i$. This shows that $x_{1} \mid p_{i}$ for all $i$ and hence $\beta D_{1} \in \mathrm{D}(\mathcal{A})$. This is a contradiction, and hence $\mathrm{D}(\mathcal{A})$ is not free.

Different arrangements can yield the same module of derivations, as the next theorem shows.

Theorem 2.6. If $\mathcal{A}$ is any arrangement, $\mathrm{H}_{1}, \ldots, \mathrm{H}_{k} \in \mathcal{A}$ and $\mathcal{B}=\mathcal{A} \cup$ $\left\{\mathrm{H}_{1} \cap \cdots \cap \mathrm{H}_{k}\right\}$ then $\mathrm{D}(\mathcal{A})=\mathrm{D}(\mathcal{B})$.

Proof. This result follows since if $\mathrm{J}=\mathrm{H}_{1} \cap \cdots \cap \mathrm{H}_{k}$ then $I_{\mathrm{J}}=I_{\mathrm{H}_{1}}+\cdots+$ $I_{\mathrm{H}_{k}}$.

Hence one could routinely assume that an arrangement is closed under intersections, as some authors do. In this paper, however, we shall not make this assumption.

\section{Generic hyperplane arrangements.}

In this section we review the case where $\mathcal{A}$ is a generic hyperplane arrangement. In particular, we find a minimal list of generators for $\mathrm{D}(\mathcal{A})$ if $\mathcal{A}$ is a generic hyperplane arrangement, and then compute the projective dimension of $\mathrm{D}(\mathcal{A})$ as an $S$-module. Much of what is found here can be gleaned from [2] and [4]. Here we give a straight-forward derivation of those results.

An essential $\ell$-arrangement $\mathcal{A}$ is generic if $\ell>1$ and every collection of $\ell$ hyperplanes from $\mathcal{A}$ is also essential. A boolean arrangement is a generic arrangement with exactly $\ell$ hyperplanes. Every 2 -arrangement is generic.

For the rest of this section we shall assume that $\mathcal{A}$ is generic. Furthermore, since each hyperplane is determined by an element of $V^{*}$ we shall describe each arrangement by listing functionals corresponding to each hyperplane. To this end we shall always choose a basis $\left\{x_{1}, \ldots, x_{\ell}\right\}$ for $V^{*}$ such that $\mathcal{A}=\left\{\alpha_{1}=x_{1}, \ldots, \alpha_{\ell}=x_{\ell}, \alpha_{\ell+1}, \ldots, \alpha_{n}\right\}$ and we let $Q=\prod \alpha_{\mathrm{H}}$ and call it a defining polynomial for $\mathcal{A}$. If $\ell<5$ we shall often use $x, y, z$ and $w$ for $x_{1}, \ldots, x_{4}$.

The following two results are well-known.

Lemma 3.1. If $\mathcal{A}$ is a 2-arrangement then $\mathrm{D}(\mathcal{A})$ is a free $S$-module with basis $\left\{\theta_{E}, \frac{Q}{x} D_{y}\right\}$. 
Lemma 3.2. If $\mathcal{A}$ is a boolean $\ell$-arrangement then $\mathrm{D}(\mathcal{A})$ is a free $S$-module with basis $\left\{x_{1} D_{1}, \ldots, x_{\ell} D_{\ell}\right\}$.

If $\ell>3$ and $\mathcal{A}$ is generic but not boolean, then $\mathrm{D}(\mathcal{A})$ is not free. To find generators of $\mathrm{D}(\mathcal{A})$ in this case we consider the intersection poset $L(\mathcal{A})$. $L(\mathcal{A})$ consists of all intersections of the elements of $V$, including the empty intersection $V$. Let $L(\mathcal{A})_{k}$ be the elements of $L(\mathcal{A})$ of dimension $k$. If $X \in L(\mathcal{A})$ let $\mathcal{A}_{X}=\{\mathrm{H} \in \mathcal{A} \mid X \subseteq \mathrm{H}\}, Q_{X}$ be a defining polynomial for $\mathcal{A}_{X}$ and $\pi_{X}=\frac{Q}{Q_{X}}$. If $X \in L(\mathcal{A})_{1}$ choose $\gamma_{X}=\sum b_{i} D_{i}$ non-zero with $b_{i} \in \mathbf{K}$ such that $\gamma_{X}\left(\alpha_{\mathrm{H}}\right)=0$ for all $\mathrm{H} \in \mathcal{A}_{X}$. This derivation may be identified with a vector parallel to $X$ and is projectively unique. Let $\theta_{X}=\pi_{X} \gamma_{X}$. One can easily see that $\theta_{X} \in \mathrm{D}(\mathcal{A})$. These derivations, together with $\theta_{E}$, will be generators of $\mathrm{D}(\mathcal{A})$. To prove this we need to introduce the concept of deletion and restriction.

Let $\mathcal{A}$ be any hyperplane arrangement and choose $\mathrm{H} \in \mathcal{A}$. Let $\mathcal{A}^{\prime}=$ $\mathcal{A} \backslash\{\mathrm{H}\}$ and call it the deletion of $\mathcal{A}$ with respect to $\mathrm{H}$. Let $\mathcal{A}^{\prime \prime}$ be the hyperplane arrangement in $\mathrm{H}$ with hyperplanes $\left\{\mathrm{H}^{\prime} \cap \mathrm{H} \mid \mathrm{H}^{\prime} \in \mathcal{A}^{\prime}\right\}$ and call it the restriction of $\mathcal{A}$ to $\mathrm{H}$. $\left(\mathcal{A}, \mathcal{A}^{\prime}, \mathcal{A}^{\prime \prime}\right)$ is called a triple of arrangements.

If $\mathcal{A}$ is generic and non-boolean then $\mathcal{A}^{\prime}$ is also generic. If $\ell>2$ and $\mathcal{A}$ is generic then $\mathcal{A}^{\prime \prime}$ is also generic. Furthermore, if $\mathcal{A}$ is generic then $L\left(\mathcal{A}^{\prime \prime}\right)$ may be identified with those elements of $L(\mathcal{A})$ contained in $\mathrm{H}$.

Next, we recall the short exact sequence of [1, Prop. 4.45]. If we choose coordinates for $V$ so that the functional associated with $\mathrm{H}$ is $x_{1}$, then multiplication by $x_{1}$ yields an injective homomorphism $\mu: \mathrm{D}\left(\mathcal{A}^{\prime}\right) \rightarrow \mathrm{D}(\mathcal{A})$. We can also restrict derivations to $\mathrm{H}$. We identify the coordinate ring of $\mathrm{H}$ with $S^{\prime \prime}=\mathbf{K}\left[x_{2}, \ldots, x_{\ell}\right]$. The canonical surjection $S \rightarrow S^{\prime \prime}$ then provides an $S$-module structure for $\mathrm{D}\left(\mathcal{A}^{\prime \prime}\right)$. If $\theta \in \mathrm{D}(\mathcal{A})$ and $f \in S^{\prime \prime}$ then let $r(\theta)(f)=\left.\theta(f)\right|_{x_{1}=0}$. It was shown in $[\mathbf{1}]$ that $r(\mathrm{D}(\mathcal{A})) \subseteq \mathrm{D}\left(\mathcal{A}^{\prime \prime}\right)$ and that the sequence $0 \rightarrow \mathrm{D}\left(\mathcal{A}^{\prime}\right) \rightarrow \mathrm{D}(\mathcal{A}) \rightarrow \mathrm{D}\left(\mathcal{A}^{\prime \prime}\right)$ is an exact sequence of $S$-modules. We shall show that the last map is surjective in the case of generic arrangements, and in the process find a minimal generating set of $\mathrm{D}(\mathcal{A})$.

Let $\mathcal{A}$ be a hyperplane arrangement and fix $\mathrm{K} \in \mathcal{A}$. Let $F(\mathcal{A})$ denote the submodule of $\mathrm{D}(\mathcal{A})$ generated by $\theta_{E}$ and $\left\{\theta_{X} \mid X \in L(\mathcal{A})_{1}, X \subseteq \mathrm{K}\right\}$ and $F\left(\mathcal{A}^{\prime \prime}\right)$ the submodule of $\mathrm{D}\left(\mathcal{A}^{\prime \prime}\right)$ generated by $\theta_{E}^{\prime \prime}=r\left(\theta_{E}\right)$ and $\left\{\theta_{X^{\prime \prime}} \mid X^{\prime \prime} \in\right.$ $\left.L\left(\mathcal{A}^{\prime \prime}\right)_{1}, X^{\prime \prime} \subseteq \mathrm{K} \cap \mathrm{H}\right\}$.

Lemma 3.3. Let $\mathcal{A}$ be a generic arrangement and $\mathrm{K} \in \mathcal{A}$. If $\mathrm{H} \in \mathcal{A}$ and $\mathrm{H} \neq \mathrm{K}$ then $r(F(\mathcal{A}))=F\left(\mathcal{A}^{\prime \prime}\right)$.

Proof. The $\theta_{X}$ with $X \subseteq \mathrm{K}$ fall into two categories. If $X \nsubseteq \mathrm{H}$ then $x_{1} \mid \pi_{X}$ and so $r\left(\theta_{X}\right)=0$. If $X \subseteq \mathrm{H}$ then $\mathrm{H} \in \mathcal{A}_{X}$ and $x_{1}$ is not a factor of $\pi_{X}$. If $X^{\prime \prime}=\cap\left\{\mathrm{J} \mid \mathrm{J} \in \mathcal{A}_{X}\right\}$ then $X^{\prime \prime} \in L\left(A^{\prime \prime}\right)$ and since $\mathcal{A}$ and $\mathcal{A}^{\prime \prime}$ are generic, one can see that $r\left(\theta_{X}\right)=\theta_{X^{\prime \prime}}$. Furthermore, since $\mathcal{A}$ is generic, each element of $L\left(A^{\prime \prime}\right)$ containing $\mathrm{K} \cap \mathrm{H}$ arises in this fashion; and hence, $r$ is surjective. 
Theorem 3.4. If $\mathcal{A}$ is a non-boolean generic $\ell$-arrangement then

$$
0 \longrightarrow \mathrm{D}\left(\mathcal{A}^{\prime}\right) \stackrel{\mu}{\longrightarrow} \mathrm{D}(\mathcal{A}) \stackrel{r}{\longrightarrow} \mathrm{D}\left(\mathcal{A}^{\prime \prime}\right) \longrightarrow 0
$$

is a short exact sequence of $S$-modules. Furthermore, for any $\mathrm{K} \in \mathcal{A}, \mathrm{D}(\mathcal{A})$ is generated by $\theta_{E} \cup\left\{\theta_{X} \mid X \in L(\mathcal{A})_{1}, X \subseteq \mathrm{K}\right\}$.

Proof. We shall prove the theorem by induction on $\ell$. The base case $\ell=2$ is Lemma 3.1. Now let $\ell>2$. We shall prove this by induction on the number of hyperplanes of $\mathcal{A}$. For this inner induction, we shall prove the base case together with the inductive step.

Let $\mathcal{A}$ be a non-boolean generic $\ell$-arrangement. Since $\mathcal{A}$ is generic and non-boolean, $\mathcal{A}^{\prime}$ and $\mathcal{A}^{\prime \prime}$ are generic and by induction, Lemma 3.1 or Lemma 3.2, have generators listed by the theorem. Since

$$
\mathrm{D}\left(\mathcal{A}^{\prime \prime}\right)=r(F(\mathcal{A})) \subseteq r(\mathrm{D}(\mathcal{A})) \subseteq \mathrm{D}\left(\mathcal{A}^{\prime \prime}\right),
$$

we see that $r$ is surjective.

Now suppose that $\theta \in \mathrm{D}(\mathcal{A})$. Since $\mathrm{D}\left(\mathcal{A}^{\prime \prime}\right)=F\left(\mathcal{A}^{\prime \prime}\right)$ we can, by Lemma 3.3, choose $\eta \in F(\mathcal{A})$ so that $r(\eta)=r(\theta)$. By exactness we have $\theta-\eta \in \mu\left(\mathrm{D}\left(\mathcal{A}^{\prime}\right)\right)$. But by induction $\mathrm{D}\left(\mathcal{A}^{\prime}\right)$ is generated by the forms $\theta_{X}^{\prime}$ where $X^{\prime} \subseteq \mathrm{K}$. Now $\mathcal{A}$ is generic so each $X^{\prime} \in L\left(\mathcal{A}^{\prime}\right)$ is also an element of $L(\mathcal{A})$ where we denote it by $X$. By definition we then have $x_{1} \theta_{X^{\prime}}=\theta_{X}$ and so $\mu \mathrm{D}\left(\mathcal{A}^{\prime}\right) \in F(\mathcal{A})$ and hence $\theta \in F(\mathcal{A})$.

The above theorem shows that if $|\mathcal{A}|=n$ and $\mathcal{A}$ is generic, then at most $\left(\begin{array}{c}n-1 \\ \ell-2\end{array}\right)+1$ generators are needed. The short exact sequence given above allows us to compute the projective dimension of $\mathrm{D}(\mathcal{A})$.

Corollary 3.5. If $\mathcal{A}$ is generic and non-boolean then the projective dimension of $\mathrm{D}(\mathcal{A})$ as an $S$-module is $\ell-2$.

Proof. We proceed by induction on $\ell$. If $\ell=2$ then $\mathrm{D}(\mathcal{A})$ is free, so the result holds. Now assume $\ell>2$. We proceed by induction on the number of hyperplanes in $\mathcal{A}$. If $|\mathcal{A}|=\ell+1$ then $\mathcal{A}^{\prime}$ is boolean and so $\mathrm{D}\left(\mathcal{A}^{\prime}\right)$ is free. Now consider the exact sequence of Theorem 3.4. $\mathcal{A}^{\prime \prime}$ is generic and non-boolean, thus $\operatorname{pdim}_{S^{\prime \prime}} \mathrm{D}\left(\mathcal{A}^{\prime \prime}\right)=\ell-3$. But $S^{\prime \prime} \simeq S / \alpha_{\mathrm{H}} S$ and hence, by [3, Theorem 4.3.3], $\operatorname{pdim}_{S} \mathrm{D}\left(\mathcal{A}^{\prime \prime}\right)=\ell-2$. As a result, by [3, Exercise 4.1.2], if $\ell \neq 3$ we have $\operatorname{pdim}_{S} \mathrm{D}(\mathcal{A})=\ell-2$. If $\ell=3$ then our arrangement is that of [1, Example 4.34], which was shown to not be free, which implies that $\operatorname{pdim}_{S} \mathrm{D}(\mathcal{A})$ in this case is also $\ell-2$.

If $|\mathcal{A}|>\ell+1$ then $\operatorname{pdim}_{S} \mathrm{D}\left(\mathcal{A}^{\prime}\right)=\operatorname{pdim}_{S} \mathrm{D}\left(\mathcal{A}^{\prime \prime}\right)=\ell-2$ and again $[\mathbf{3}$, Exercise 4.1.2] shows that $\operatorname{pdimD}(\mathcal{A})=\ell-2$.

[4] provides a minimal projective resolution of $\mathrm{D}(\mathcal{A})$ which also shows that the minimal number of generators of $\mathrm{D}(\mathcal{A})$ is exactly $\left(\begin{array}{c}n-1 \\ \ell-2\end{array}\right)+1$. 


\section{Arrangements arising from $L(\mathcal{A})$.}

In this section we discuss arrangements which consist of a subset of $L(\mathcal{A})$ for a hyperplane arrangement $\mathcal{A}$. In particular, choose $k \geq 2$ and let $\mathcal{A}_{k}$ be the $(\ell, k)$-arrangement consisting of those elements of $L(\mathcal{A})$ of codimension $k$. Note that $\mathcal{A}_{1}=\mathcal{A}$. The next result gives a filtration which may be an interesting object of study.

Theorem 4.1. If $\mathcal{A}$ is a hyperplane arrangement, then

$$
\mathrm{D}(\mathcal{A}) \subseteq \mathrm{D}\left(\mathcal{A}_{2}\right) \subseteq \cdots \subseteq \mathrm{D}\left(\mathcal{A}_{\ell}\right)
$$

If $\mathcal{A}$ is essential then $\mathrm{D}\left(\mathcal{A}_{\ell}\right)=S^{+} \operatorname{Der}_{\mathbf{K}}(S)$.

Proof. If $\theta \in \mathrm{D}(\mathcal{A})_{k}$ then $\theta(I(X)) \subseteq S I(X)$ for each $X \in L(\mathcal{A})_{\ell-k}$. If $Y \in L(\mathcal{A})_{\ell-k-1}$ then $Y=X_{1} \cap X_{2}$ where $X_{1}$ and $X_{2}$ are elements of $L(\mathcal{A})_{\ell-k}$; but then $V(Y)=V\left(X_{1}\right)+V\left(X_{2}\right)$ and hence $\theta(Y) \subseteq Y$ and $\theta \in \mathrm{D}(\mathcal{A})_{k+1}$. If $\mathcal{A}$ is essential then $L(\mathcal{A})_{0}=\{0\}$ and since $V(0)=S^{+}$it is clear that $\mathrm{D}(\mathcal{A})_{\ell}=S^{+} \operatorname{Der}_{\mathbf{K}}(S)$.

Next we apply the notion of deletion and restriction to these modules. One easy result is the following:

Lemma 4.2. If $\mathcal{A}$ is an $\ell$-arrangement and $\mathrm{H} \in \mathcal{A}$ with defining functional $\alpha_{\mathrm{H}}$ then $\alpha_{\mathrm{H}} \mathrm{D}\left(\mathcal{A}_{k}^{\prime}\right) \subseteq \mathrm{D}\left(\mathcal{A}_{k}\right)$.

Next we consider the restriction map of Section 3.

Lemma 4.3. If $\mathcal{A}$ is a hyperplane arrangement, $\mathrm{H} \in \mathcal{A}$, and $\mathcal{A}^{\prime \prime}$ is the restriction of $\mathcal{A}$ to $\mathrm{H}$, then $r\left(\mathrm{D}\left(\mathcal{A}_{k}\right)\right) \subseteq \mathrm{D}\left(\mathcal{A}_{k-1}^{\prime \prime}\right)$ for every $2 \leq k \leq \ell$.

Proof. First choose coordinates so that $\alpha_{\mathrm{H}}=x_{1}=x$. We identify $S^{\prime \prime}$ with $\mathbf{K}\left[x_{2}, \ldots, x_{\ell}\right]$. Let $\theta \in \mathrm{D}\left(\mathcal{A}_{k}\right)$. If $X \in L\left(A^{\prime \prime}\right)_{\ell-k}$ then $I(X)=\left(\beta_{1}, \ldots, \beta_{k-1}\right)$ where the $\beta_{i}$ are linear functionals associated to the elements of $\mathcal{A}^{\prime \prime}$. There exist $a_{1}, \ldots, a_{k-1} \in \mathbf{K}$ such that $\alpha_{i}=a_{i} x+\beta_{i}$ are functionals defining elements of $\mathcal{A}$. But note that there exists $Y \in \mathcal{A}_{k}$ such that $I_{Y}=$ $\left(x, \alpha_{1}, \ldots, \alpha_{k-1}\right)=\left(x, \beta_{1}, \ldots, \beta_{k}\right)$ and so $\theta(I(X)) \subseteq\left(x, \beta_{1}, \ldots, \beta_{k-1}\right)$ and hence $r(\theta)(I(X))=\left.\theta(I(X))\right|_{x=0} \subseteq\left(\beta_{1}, \ldots, \beta_{k-1}\right)$. Since this holds true for all $X \in L\left(\mathcal{A}^{\prime \prime}\right)$ we see that $r(\theta) \in \mathrm{D}\left(\mathcal{A}_{k-1}^{\prime \prime}\right)$.

These two results will be used later when we find generators of $\mathrm{D}\left(\mathcal{A}_{k}\right)$ when $\mathcal{A}$ is generic. If $\mathcal{A}$ is boolean, the $\mathrm{D}\left(\mathcal{A}_{k}\right)$ are easily described. Let $V$ be a vector space of dimension $\ell$. Let us say that a subset $T$ of $V^{*}$ is generic if every subset of $T$ of size at most $\ell$ is linearly independent.

Lemma 4.4. Let $V$ be a vector space of dimension $\ell, S=S V^{*}$ and $\mathcal{A}=$ $\left\{\alpha_{1}, \ldots, \alpha_{n}\right\}$ be a generic subset of $V^{*}$. Let $Q=\prod_{i=1}^{n} \alpha_{i}$ and if $X \subseteq$ 
$\{1, \ldots, n\}$ let $\pi_{X}=\prod_{j \in X} \alpha_{j}$. For each $1 \leq k<\ell$ the ideal $\bigcap_{i_{1}<\cdots<i_{k}}\left(\alpha_{i_{1}}\right.$, $\left.\ldots, \alpha_{i_{k}}\right)$ is generated by

$$
\left\{\frac{Q}{\pi_{X}}|X \subseteq\{1, \ldots, n\},| X \mid=k-1\right\}
$$

and the ideal $\bigcap_{1<i_{2}<\cdots<i_{k}}\left(\alpha_{1}, \ldots, \alpha_{i_{k}}\right)$ is generated by

$$
\left\{\alpha_{1}\right\} \cup\left\{\frac{Q}{\alpha_{1} \pi_{X}}|X \subseteq\{2, \ldots, n\},| X \mid=k-2\right\} .
$$

Proof. Let $N=\bigcap_{2 \leq i_{2}<\cdots<i_{k}}\left(\alpha_{1}, \alpha_{i_{2}}, \ldots, \alpha_{i_{k}}\right)$ and $L=\bigcap_{2 \leq i_{1}<\cdots<i_{k}}\left(\alpha_{i_{1}}\right.$, $\left.\ldots, \alpha_{i_{k}}\right)$. Our goal is to show that $I=N \cap L$. To prove this we shall induct on $k$. If $k=1$ then the result is clear. Now assume $k>1$. To prove the inductive step we shall induct on $n$. If $n=k$, then again the result is clear. Now assume $n>k$.

Let $\phi: S \rightarrow S /\left(\alpha_{1}\right)$ and denote $\phi(f)$ by $\bar{f}$. Since $\phi$ is surjective and $\operatorname{ker}(\phi)$ is a subset of every $\left(\alpha_{1}, \alpha_{i_{2}}, \ldots, \alpha_{i_{k}}\right)$ we have

$$
\phi(N)=\bigcap \phi\left(\alpha_{1}, \alpha_{i_{2}}, \ldots, \alpha_{i_{k}}\right)=\bigcap\left(\overline{{i_{2}}_{2}}, \ldots, \overline{\alpha_{i_{k}}}\right) .
$$

Since $\mathcal{A}$ is generic $Q^{\prime \prime}=\phi\left(Q / \alpha_{1}\right)$ is square free and so, by induction on $k, \phi(N)$ is generated by the $\frac{Q^{\prime \prime}}{\pi_{X}}=\phi\left(\frac{Q}{\alpha_{1} \pi_{X}}\right)$ where $X \subseteq\{2, \ldots, n\}$ and $|X|=k-2$. This shows that

$$
N=\left(\alpha_{1}\right)+\left(\left\{\frac{Q}{\alpha_{1} \pi_{X}}|X \subseteq\{2, \ldots, n\},| X \mid=k-2\right\}\right) .
$$

Denote the first ideal on the right by $J$ and the second by $K$. By induction on $n$ we see that

$$
L=\left(\left\{\frac{Q}{\alpha_{1} \pi_{X}}|X \subseteq\{2, \ldots, n\},| X \mid=k-1\right\}\right) .
$$

Thus $\mathrm{I}$ is an intersection of the form $(J+K) \cap L$ with $K \subseteq L$ and whence $I=J \cap L+K$.

We claim that

$$
J \cap L+K=\left(\left\{\frac{Q}{\pi_{X}}|X \subseteq\{1, \ldots, n\},| X \mid=k-1\right\}\right)
$$

and denote the latter ideal by $M$. Clearly $M \subseteq I$ and $K \subseteq M$. It remains to show that $J \cap L \subseteq M$. If $g \in J \cap K$ then $g=h \alpha_{1}=\sum_{X} f_{X} \frac{Q}{\alpha_{1} \pi_{X}}$. If $Y \subseteq\{2, \ldots, n\}$ with $|Y|=k-1$ and $I_{Y}=\left(\left\{\alpha_{i} \mid i \in Y\right\}\right)$ then consider $\psi: S \rightarrow S / I_{Y}$ and again denote $\psi(f)$ by $\bar{f}$. Since $k<\ell$, this is an integral domain. Note that $\bar{g}=\bar{h} \overline{\alpha_{1}}=\overline{f_{Y}} \frac{Q}{\frac{Q}{\alpha_{1} \pi_{Y}}}$ and hence there exists $h_{Y}$ such that 
$\overline{f_{Y}}=\overline{\alpha_{1}} \overline{h_{Y}}$ and so $f_{Y}=\alpha_{1} h_{Y}+g_{Y}$ where $g_{Y} \in I_{Y}$, but then $f_{Y} \frac{Q}{\alpha_{1} \pi_{Y}} \in M$. Since this holds for all $Y$ we see that $J \cap L \subseteq M$ and the result is proven.

We can use the above result to determine generators for $\mathrm{D}\left(\mathcal{A}_{k}\right)$ if $\mathcal{A}$ is essential and boolean.

Theorem 4.5. Let $\mathcal{A}=\left\{\mathrm{H}_{1}, \ldots, \mathrm{H}_{\ell}\right\}$ be a boolean $\ell$-arrangement with $\mathrm{H}_{i}=$ $V\left(x_{i}\right)$ and let $Q=\prod_{i=1}^{\ell} x_{i}$ then, for each $k>1, \mathrm{D}\left(\mathcal{A}_{k}\right)$ is generated as an $S$-module by

$$
\left\{x_{i} D_{i}\right\}_{i=1}^{\ell} \bigcup\left\{\frac{Q}{Q_{X}} D_{i} \mid X \in L(\mathcal{A})_{\ell-k+1}, X \subseteq \mathrm{H}_{i}, 1 \leq i \leq \ell\right\} .
$$

Proof. Let $\theta \in \mathrm{D}\left(\mathcal{A}_{k}\right)$ and write $\theta=\sum p_{i} D_{i}$. Choose $1 \leq j \leq \ell$ and let $J=\{1, \ldots, n\} \backslash\{j\}$. Now $p_{j}=\theta\left(x_{j}\right) \in \bigcap_{\substack{Y \subseteq J \\|Y|=k-1}}\left(\alpha_{j},\left\{\alpha_{m}\right\}_{m \in Y}\right)=$ $\left(\alpha_{j},\left\{\frac{Q}{\alpha_{j} \pi_{Y}}|Y \subseteq J| Y \mid,=k-2\right\}\right)$. But each $\alpha_{j} \pi_{Y}$ is the defining polynomial of a subarrangement of $\mathcal{A}$ of size $k-1$ corresponding to an element of $L(\mathcal{A})$ of dimension $\ell-k+1$. The result follows.

Note that as $k$ increases the modules pick up smaller and smaller "factors" of the $Q D_{i}$. This pattern will also hold for generic arrangements. The above result also allows us to compute the projective dimensions of $\mathrm{D}\left(\mathcal{A}_{2}\right)$ for a boolean arrangement.

Theorem 4.6. If $\mathcal{A}$ is boolean then the $\operatorname{pdim}_{S}\left(\mathrm{D}\left(\mathcal{A}_{2}\right)\right)=1$.

Proof. It suffices to consider the case where $\mathcal{A}$ is essential. Let $P_{0}$ be the free $S$-module with generators $\alpha_{1}, \ldots, \alpha_{\ell}, \beta_{1}, \ldots, \beta_{\ell}$. Let $\psi: P_{0} \rightarrow \mathrm{D}(\mathcal{A})$ be the map define by $\psi\left(\alpha_{i}\right)=x_{i} D_{i}$ and $\psi\left(\beta_{i}\right)=\frac{Q}{x_{i}} D_{i}$. We will show that ker $\psi$ is a free $S$-module.

Suppose $\psi\left(\sum f_{i} \alpha_{i}+\sum g_{i} \beta_{i}\right)=0$, then for each $i$ we have $x_{i} f_{i}+\frac{Q}{x_{i}} g_{i}=0$. Thus there exists $h_{i}$ so that $f_{i}=\frac{Q}{x_{i}} h_{i}$ and $g_{i}=-x_{i} h_{i}$. Thus ker $\psi=$ $\left\{\sum\left(\frac{Q}{x_{i}} \alpha_{i}-x_{i} \beta_{i}\right) h_{i} \mid h_{i} \in S\right\}$ is a free $S$-module of rank $\ell$.

\section{Generic $(l, k)$ arrangements.}

In this section we assume that $\mathcal{A}$ is a generic $\ell$-arrangement and work with the associated $(\ell, k)$ arrangements. We begin by finding generating sets for $\mathrm{D}\left(\mathcal{A}_{2}\right)$ using elements of $L(\mathcal{A})$. It is useful to consider some examples of $(\ell, 2)$-arrangements arising in this fashion. It is convenient to describe the subspaces using their corresponding ideals.

Example 5.1. In $\mathbf{K}^{3}$ let $\mathcal{A}=\{x, y, z, x+y+z\}$, so that

$$
\mathcal{A}_{2}=\{(x, y),(x, z),(x, x+y+z),(y, z),(y, x+y+z),(z, x+y+z)\} .
$$


$\mathrm{D}\left(\mathcal{A}_{2}\right)$ has generators

$$
\theta_{E}, \quad y z(x+y+z) D_{x}, \quad x y\left(D_{x}-D_{y}\right), \quad x y z D_{z}
$$

$y(x+y+z) D_{y}, \quad x z(x+y+z) D_{y}, \quad z(x+y+z) D_{z}, \quad x y(x+y+z) D_{z}$.

In $\mathbf{K}^{3}$ if $\mathcal{B}=\{x, y, x+y, z, x+y+z\}$ then $\mathrm{D}(\mathcal{B})$ is a free $S$-module but $\mathcal{B}_{2}$ is the same arrangement as $\mathcal{A}_{2}$ listed above. Hence a free arrangement and a generic arrangement may yield identical $(\ell, 2)$-arrangements.

Example 5.2. In $\mathbf{K}^{4}$ let $\mathcal{A}=\{w, x, y, z, w+x+y+z\}$, so that

$$
\begin{gathered}
\mathcal{A}_{2}=\{(w, x),(w, y),(w, z),(w, w+x+y+z),(x, y), \\
(x, z),(x, w+x+y+z),(y, z),(y, w+x+y+z),(z, w+x+y+z)\}
\end{gathered}
$$

$\mathrm{D}\left(\mathcal{A}_{2}\right)$ has generators

$$
\begin{gathered}
\theta_{E}, \quad x y z(w+x+y+z) D_{w}, \quad w y z(w+x+y+z) D_{x} \\
w x z(w+x+y+z) D_{y}, \quad w x y(w+x+y+z) D_{z}, \quad x y\left(D_{x}-D_{y}\right) \\
y z\left(D_{y}-D_{z}\right), \quad w x\left(D_{w}-D_{x}\right), \quad x y z w D_{y} \\
y(w+x+y+z) D_{y}, \quad z(w+x+y+z) D_{z} .
\end{gathered}
$$

These examples motivate the following definition. As usual, choose a basis $\left\{x_{1}, \ldots, x_{\ell}\right\}$ of $V^{*}$. Let $\mathrm{H} \in \mathcal{A}$ with $\alpha_{\mathrm{H}}$ its defining functional and write $\alpha_{\mathrm{H}}=\sum a_{i} x_{i}$. Let $\eta_{\mathrm{H}}=\frac{Q}{\alpha_{\mathrm{H}}} \sum a_{i} D_{x_{i}}$. One can easily see that $\eta_{\mathrm{H}} \in \mathrm{D}\left(\mathcal{A}_{2}\right)$. Our goal is to show that the $\theta_{X}$ and $\eta_{\mathrm{H}}$ together with $\theta_{E}$ generate $\mathrm{D}\left(\mathcal{A}_{2}\right)$ for a generic arrangement.

To prove this we will induct on the number of hyperplanes of $\mathcal{A}$ using the method of deletion and restriction. An examination of the examples listed above leads us to the following lemma.

Lemma 5.3. If $\mathcal{A}$ is generic, then $r: \mathrm{D}\left(\mathcal{A}_{2}\right) \rightarrow \mathrm{D}\left(\mathcal{A}^{\prime \prime}\right)$ is surjective.

Proof. The result is clear if $\ell=2$. If $\ell>2$ and $\mathcal{A}$ is generic, then so is $\mathcal{A}^{\prime \prime}$. One then notes that $\left\{r\left(\theta_{X}\right)\right\}_{X \in L(\mathcal{A})_{1}} \cup r\left(\theta_{E}\right)$ is the generating set of $D\left(\mathcal{A}^{\prime \prime}\right)$ given in Theorem 3.4.

We can now state one of our main results:

Theorem 5.4. Let $\mathcal{A}$ be a generic $\ell$-arrangement with $\ell>2$ and $\mathrm{H} \in \mathcal{A}$. The sequence

$$
\mathrm{D}\left(\mathcal{A}_{2}^{\prime}\right) \oplus S \stackrel{\phi}{\rightarrow} \mathrm{D}\left(\mathcal{A}_{2}\right) \stackrel{r}{\rightarrow} \mathrm{D}\left(\mathcal{A}^{\prime \prime}\right) \rightarrow 0
$$

is exact where $\phi(\theta, f)=\alpha_{\mathrm{H}} \theta+f \eta_{\mathrm{H}}$. 
Proof. Since $r\left(\eta_{\mathrm{H}}\right)=0$ we only need to show that $\operatorname{ker}(r) \subseteq \operatorname{im} \phi$. Let $\mathcal{A}=\left\{\mathrm{H}, \mathrm{H}_{2}, \ldots, \mathrm{H}_{n}\right\}$ and choose coordinates so $\mathrm{H}=V\left(x_{1}\right)$. Let $\theta \in \operatorname{ker}(r)$ and write $\theta=\sum_{i=1}^{\ell} p_{i} D_{i}$. Since $r(\theta)=0, p_{i}=x_{i} q_{i}$ for $i>1$.

Since $\mathcal{A}$ is generic the ideals $\left\{\left(x_{1}, \alpha_{i}\right)\right\}_{i>1}$ are distinct, hence $\theta\left(x_{1}\right) \in$ $\cap_{i=2}^{n}\left(x_{1}, \alpha_{i}\right)=\left(x_{1}, \prod_{i=2}^{n} \alpha_{i}\right)=\left(x_{1}, \frac{Q}{x_{1}}\right)$. So write $p=x_{1} q_{1}+\frac{Q}{x_{1}} s$, and $\tau=\sum_{i=1}^{\ell} q_{i} D_{x_{i}}$ so that $\theta=x_{1} \tau+s \eta_{X}$. It suffices to show $\tau \in \mathrm{D}\left(\mathcal{A}_{2}^{\prime}\right)$. Let $X \in L\left(\mathcal{A}^{\prime}\right)_{2}$, then since $\frac{Q}{x_{1}} \in I_{X}$ we see that $\theta\left(I_{X}\right) \subset I_{X}$ iff $x_{1} \mu\left(I_{X}\right) \subseteq I_{X}$. But since $x_{1} \notin I_{X}$ (as $\mathcal{A}$ is generic), we see that $x_{1} \mu\left(I_{X}\right) \subseteq I_{X}$ iff $\mu\left(I_{X}\right) \subseteq$ $I_{X}$, hence $\mu \in \mathrm{D}\left(\mathcal{A}_{2}^{\prime}\right)$.

With the above exact sequence we can prove the following result.

Theorem 5.5. Let $\mathcal{A}$ be a generic arrangement, $\ell>2$ and $K \in \mathcal{A} . \mathrm{D}\left(\mathcal{A}_{2}\right)$ is generated by $\left\{\theta_{E}\right\} \cup\left\{\theta_{X} \mid X \in L(\mathcal{A})_{1}, X \subseteq K\right\} \cup\left\{\eta_{\mathrm{H}} \mid \mathrm{H} \in \mathcal{A}\right\}$.

Proof. The proof here is very similar to that of Theorem 3.4. We induct on $\ell$. We shall prove the base case together with the inductive step. To show these we induct on $|\mathcal{A}|$. If $\mathcal{A}$ is boolean then the result follows from Theorem 4.5. If $|\mathcal{A}|>\ell$ then we use the exact sequence of the previous theorem. If $\theta \in \mathrm{D}\left(\mathcal{A}_{2}\right)$ then since $r$ is surjective and $\mathcal{A}^{\prime \prime}$ is generic we can choose $\eta \in \tilde{\mathrm{D}}(\mathcal{A})$ so that $r(\eta)=r(\theta)$. Hence $\theta-\eta$ is in the image of $\phi$. But $\mathcal{A}^{\prime}$ is generic or boolean, so by induction its generators are given by the theorem. As in the proof of Theorem 3.4 one sees that when the generators of $\mathrm{D}\left(\mathcal{A}^{\prime}\right)$ are multiplied by $\alpha_{\mathrm{H}}$ they become the generators postulated for $\mathrm{D}(\mathcal{A})$. The result follows.

Now assume $k>2$. For each $\mathrm{H} \in \mathcal{A}$ write $\alpha_{\mathrm{H}}=\sum a_{i} x_{i}$. Now let $Y \in L(\mathcal{A})_{\ell-k+1}$ with $Y \subseteq \mathrm{H}$ and let $\eta_{\mathrm{H}, Y}=\frac{Q}{\pi_{Y}} \sum a_{i} D_{x_{i}}$. Let $F\left(\mathcal{A}_{k}\right)$ be the $S$-submodule of $\mathrm{D}\left(\mathcal{A}_{k}\right)$ generated by $\theta_{E}$ together with the $\theta_{X}$ and the set of all $\eta_{\mathrm{H}, Y}$.

The rest of this section is devoted to the proof of the following theorem.

Theorem 5.6. Let $\ell>2, \mathcal{A}$ be a generic $\ell$-arrangement, $2 \leq k<\ell$ and $\mathrm{K} \in \mathcal{A} . \mathrm{D}\left(\mathcal{A}_{k}\right)$ is generated as an $S$-module by

$$
\begin{aligned}
\left\{\theta_{E}\right\} \cup\left\{\theta_{X} \mid X \in L(\mathcal{A})_{1}, X \subseteq K\right\} & \subseteq\left\{\eta_{\mathrm{H}, Y} \mid \mathrm{H} \in \mathcal{A}, Y \in L(\mathcal{A})_{\ell-k+1}, Y \subseteq \mathrm{H}\right\} .
\end{aligned}
$$

We shall prove this by induction on $\ell$. The base case $\ell=3$ is Theorem 5.5. Now assume $\ell>3$. To prove this we induct on $k$. The case $k=2$ is also Theorem 5.5, so assume $k>2$. To show this we induct on the number of hyperplanes of $\mathcal{A}$. If $|\mathcal{A}|=\ell$, then the result follows from Theorem 4.5. Hence, we assume that $|\mathcal{A}|>\ell$.

We begin by choosing $\mathrm{H} \in \mathcal{A}$ with $\mathrm{H} \neq \mathrm{K}$, and choose coordinates so that $\alpha_{\mathrm{H}}=x_{1}$. Let $\mathcal{A}^{\prime}$ be the deletion of $\mathcal{A}$ with respect to $\mathrm{H}$ and $\mathcal{A}^{\prime \prime}$ 
the restriction of $\mathcal{A}$ to $\mathrm{H}$. Let $F\left(\mathcal{A}_{k}\right)$ be the $S$-module generated by the derivations given in the theorem. Our goal is to show that $F\left(\mathcal{A}_{k}\right)=\mathrm{D}\left(\mathcal{A}_{k}\right)$.

Lemma 5.7. If $\mathcal{A}$ is generic, then $r: F\left(\mathcal{A}_{k}\right) \rightarrow \mathrm{D}\left(\mathcal{A}_{k-1}^{\prime \prime}\right)$ is surjective.

Proof. Since $\mathcal{A}$ is generic, so is $\mathcal{A}^{\prime \prime}$ and, by induction on $\ell, \mathrm{D}\left(\mathcal{A}_{k-1}^{\prime \prime}\right)$ has generators given in the theorem. One then notes that restriction of the generators of $F\left(\mathcal{A}_{k}\right)$ are either zero or precisely the generators given for $\mathrm{D}\left(\mathcal{A}_{k-1}^{\prime \prime}\right)$. In particular, let $\mathrm{H}^{\prime \prime} \in \mathcal{A}^{\prime \prime}$ and $Y^{\prime \prime} \in L\left(\mathcal{A}^{\prime \prime}\right)_{\ell-k+1}$ then there is a unique $Y \in L(\mathcal{A})$ so that $Y=Y^{\prime \prime}$. Since $Y \subset \mathrm{H}$ we have $x_{1} \mid \pi_{Y}$. Now $\eta_{\mathrm{H}, Y}=\frac{Q}{\pi_{Y}} \sum_{k=1}^{\ell} a_{i} D_{i}=\frac{Q / \alpha_{\mathrm{H}}}{\pi_{Y} / \alpha_{\mathrm{H}}} \sum_{i=1}^{\ell} a_{i} D_{i}$ and since $\left.\frac{Q}{\alpha_{\mathrm{H}}}\right|_{x_{1}=0}=Q^{\prime \prime}$ and $\left.\frac{\pi_{Y}}{\alpha_{\mathrm{H}}}\right|_{x_{1}=0}=\pi_{Y^{\prime \prime}}$ we see that $r\left(\eta_{\mathrm{H}, Y}\right)=\frac{Q^{\prime \prime}}{\pi_{Y^{\prime \prime}}} \sum_{i=2}^{\ell} a_{i} D x_{i}=\eta_{\mathrm{H}^{\prime \prime}, Y^{\prime \prime}}$.

Lemma 5.8. Let $\mathcal{A}$ be a generic arrangement and $\mathrm{H} \in \mathcal{A}$, then the sequence

$$
\mathrm{D}\left(\mathcal{A}_{k}^{\prime}\right) \bigoplus_{\substack{Y \in L(\mathcal{A})_{k-1} \\ Y \subseteq \mathrm{H}}} S \stackrel{\phi}{\longrightarrow} \mathrm{D}\left(\mathcal{A}_{k}\right) \stackrel{r}{\longrightarrow} \mathrm{D}\left(\mathcal{A}_{k-1}^{\prime \prime}\right) \longrightarrow 0
$$

is exact where $\phi\left(\theta,\left(f_{Y}\right)\right)=\alpha_{\mathrm{H}} \theta+\sum_{Y} f_{Y} \eta_{\mathrm{H}, Y}$.

Proof. We only need to show that $\operatorname{ker}(r) \subseteq \operatorname{im} \phi$. Let $\mathcal{A}=\left\{\mathrm{H}, \mathrm{H}_{2}, \ldots, \mathrm{H}_{n}\right\}$ and choose coordinates so that $\mathrm{H}=V\left(x_{1}\right)$. Let $\theta \in \operatorname{ker}(r)$ and write $\theta=$ $\sum_{i=1}^{\ell} p_{i} D_{i}$. If $r(\theta)=0$ then $p_{i}=x_{1} q_{i}$ for each $i>1$.

Since $\mathcal{A}$ is generic the ideals $\left\{\left(x_{1}, \alpha_{i_{2}}, \ldots, \alpha_{i_{k}}\right) \mid 2 \leq i_{2}<\cdots<i_{k}\right\}$ are distinct, hence by Lemma 4.4

$$
\begin{aligned}
& \theta\left(x_{1}\right) \in \bigcap_{2 \leq i_{2}<\cdots<i_{k}}\left(x_{1}, \alpha_{i_{2}}, \ldots \alpha_{i_{k}}\right) \\
& =\left(x_{1},\left\{\frac{Q}{x_{1} \pi_{X}}|X \subset\{2, \ldots n\}| X \mid=k-2\right\}\right) .
\end{aligned}
$$

Write $p_{1}=x_{1} q_{1}+\sum_{X} s_{X} \frac{Q}{x_{1} \pi_{X}} s$, and let $\tau=\sum_{i=1}^{\ell} q_{i} D_{i}$ so that $\theta=x_{1} \tau+$ $\sum s_{X} \eta_{X}$. Hence, it suffices to show $\tau \in \mathrm{D}\left(\mathcal{A}_{k}^{\prime}\right)$. Let $Y \in L\left(\mathcal{A}^{\prime}\right)_{k}$, then since $\frac{Q}{x_{1} \pi_{X}} \in I_{Y}$ for each $X \neq Y$ we see that $\theta\left(I_{Y}\right) \subset I_{Y}$ iff $x_{1} \tau\left(I_{Y}\right) \subseteq I_{Y}$. But since $x_{1} \notin I_{Y}$ (as $\mathcal{A}$ is generic), we see that $x_{1} \tau\left(I_{Y}\right) \subseteq I_{Y}$ iff $\tau\left(I_{Y}\right) \subseteq I_{Y}$, hence $\tau \in \mathrm{D}\left(\mathcal{A}_{k}^{\prime}\right)$.

With the above exact sequence we can finish the proof our main result. If $\theta \in \mathrm{D}\left(\mathcal{A}_{k}\right)$ then choose $\eta \in F\left(\mathcal{A}_{k}\right)$ so that $r(\theta)=r(\eta)$, in which case $\theta-\eta \in \operatorname{im} \phi$. But by induction $\mathrm{D}\left(\mathcal{A}_{k}^{\prime}\right)=F\left(A_{k}^{\prime}\right)$ and since $\phi$ sends the generators of $\mathrm{D}\left(\mathcal{A}_{k}^{\prime}\right)$ to generators of $F\left(\mathcal{A}_{k}\right)$, we see that $\theta \in F\left(\mathcal{A}_{k}\right)$.

An interesting result would be to compute minimal resolutions of these modules. 


\section{References}

[1] P. Orlik and H. Terao, Arrangements of Hyperplanes, Springer-Verlag, 1992.

[2] L. Rose and H. Terao, A free resolution of the module of logarithmic forms of a generic arrangement, J. of Algebra, 136 (1991), 376-400.

[3] C. Weibel, An Introduction to Homological Algebra, Cambridge University Press, 1994.

[4] S. Yuzvinsky, A free resolution of the module of derivations for generic arrangement, J. of Algebra, 136 (1991), 432-438.

Received February 14, 1999.

Department of Mathematical Sciences

University of Alaska Fairbanks

FAIRBANKs, AK 99775

E-mail address: ffjjw@uaf.edu 